\title{
A novel approach to medical radioisotope production using inverse kinematics
}

\author{
Márcia Regina Dias Rodrigues ${ }^{1 *}$, Victor E. Iacob ${ }^{1}$, Ninel Nica ${ }^{1}$, Brian Roeder ${ }^{1}$, Gabriel Tabacaru ${ }^{1}$, Kang Wang ${ }^{1}$, \\ Meixiang $\mathrm{Yu}^{4}$, Paulo Zanotti-Fregonara ${ }^{4}$, Justin Mabiala ${ }^{5}$, Jedidiah Romo ${ }^{1}$, Georgios A. Souliotis ${ }^{2}$ and Aldo \\ Bonasera $^{1,3}$. \\ ${ }^{1}$ Cyclotron Institute, Texas A\&M University, USA \\ ${ }^{2}$ Laboratory of Physical Chemistry, National and Kapodistrian University of Athens, Greece \\ ${ }^{3}$ Laboratori Nazionali del Sud, INFN, Italy \\ ${ }^{4}$ Houston Methodist Research Institute, USA \\ ${ }^{5}$ Prairie View A\&M University, Prairie View, USA
}

\begin{abstract}
The inverse kinematics methodology using a gas target has been applied to produce medically important radionuclides at the Cyclotron Institute at Texas A\&M University. The production of the theranostic radionuclide ${ }^{67} \mathrm{Cu}\left(\mathrm{T}_{1 / 2}=62 \mathrm{~h}\right)$ through the reaction of a ${ }^{70} \mathrm{Zn}$ beam at $15 \mathrm{MeV} /$ nucleon with a hydrogen gas target was performed. The activities at end of irradiation and the thick target yield were obtained for ${ }^{67} \mathrm{Cu}$. A test using the forward-focused neutrons from the primary reaction to irradiate nat $\mathrm{Zn}$ to produce ${ }^{67} \mathrm{Cu}$ is also presented.
\end{abstract}

\section{Introduction}

A novel approach to produce medically important radionuclides using inverse kinematics has been developed at the Cyclotron Institute at Texas A\&M University. A heavy-ion beam is accelerated at an appropriate energy and focused on a light gas target. A foil catcher is positioned after the target to collect the isotopes of interest, typically emitted in a narrow angle along with the beam direction. Secondary emitted particles such as neutrons from the primary nuclear reaction can be used to irradiate other targets for further radionuclide production. As the quantity of material required to prepare heavy-ion beam is less than that used in the standard solid target approach, material costs are expected to be considerably reduced through this methodology. A detailed discussion about the methodology can be found in [1].

Medical radionuclides are essential in nuclear medicine in the fields of diagnostic imaging and radioimmunotherapy (RIT). RIT in nuclear medicine involves the injection of a radioisotope-labeled monoclonal antibody (mAb) to treat cancer. The radionuclides are carried directly toward the tumor and the emitted radiation breaks the DNA inducing the carcinogenic cell death. Radionuclides emitting lowrange highly ionizing radiation are essential for radioimmunotherapy. The longest-lived radioisotope of copper, ${ }^{67} \mathrm{Cu}$, is ideal for both radioimmunotherapy and imaging, i.e. radioimmunotheranostics [2], for several reasons. First, from a chemical perspective, copper is an essential trace element that can be linked to antibodies, proteins and other biologically important molecules. The half-life of ${ }^{67} \mathrm{Cu}(62 \mathrm{~h})$ is appropriate to deliver a high dose rate to the tumor. While the $\beta^{-}$decay $\left(\mathrm{E}_{\max }=\right.$ $562 \mathrm{keV}$ ) is responsible for the therapeutic part, the emission of the $\gamma$ radiation of $185 \mathrm{keV}(48.7 \%)$ and 93 $\mathrm{keV}(16.1 \%)$ is for the diagnostics. The ${ }^{67} \mathrm{Cu}$ is suitable for imaging the radiotracer distribution by single-photon emission computerized tomography (SPECT) and it can be combined with the same type of radiopharmaceuticals as ${ }^{64} \mathrm{Cu}\left(\mathrm{T}_{1 / 2}=12.7 \mathrm{~h}\right)$ or ${ }^{61} \mathrm{Cu}$ $\left(\mathrm{T}_{1 / 2}=3.3 \mathrm{~h}\right)$, leading to efficient theranostic pairs. The ${ }^{67} \mathrm{Cu}$ is also responsible to the theranostic approach to pretargeted radioimmunotherapy (PRIT) [3]. The main factor limiting preclinical and clinical use is its limited availability [4].

The production of ${ }^{67} \mathrm{Cu}$ has started in the 1960 s using nuclear reactors and continues until the present. Recently the interest has shifted towards production at particle accelerators. The main production route is via the reaction ${ }^{68} \mathrm{Zn}(\mathrm{p}, 2 \mathrm{p}){ }^{67} \mathrm{Cu}[5-8]$ that makes use of intense medium-energy $\left(E_{p}=70-100 \mathrm{MeV}\right)$ proton beams. Another production route based on lower-energy charged particle reactions is ${ }^{70} \mathrm{Zn}(\mathrm{p}, \alpha)^{67} \mathrm{Cu}[9-11]$. The present work uses as primary reaction the inverse kinematic reaction $\mathrm{p}\left({ }^{70} \mathrm{Zn},{ }^{67} \mathrm{Cu}\right) \alpha$ and the secondary reaction as ${ }^{n a t} \mathrm{Zn}(\mathrm{n}, \mathrm{x})^{67} \mathrm{Cu}$. The structure of the paper is as follows: in section 2 the experimental setup and the measurements are described. In section 3, the details of the analysis and results are presented including the thick target yields and the use of the neutrons for secondary isotope production. Finally, conclusions and future plans are presented in section 4 .

\footnotetext{
* Corresponding author: mrodrigues@tamu.edu
} 


\section{Experimental measurements}

set

up

and

\subsection{Irradiation set up}

The ${ }^{70} \mathrm{Zn}^{15+}$ beam was produced by the ECR source and accelerated by the K500 superconducting cyclotron at the Cyclotron Institute at Texas A\&M University to an energy of $15 \mathrm{MeV} /$ nucleon, then transported to the target chamber of the MARS recoil separator [12]. The beam impinged on a cryogenic gas cell filled with $\mathrm{H}_{2}$ gas at a pressure of 2.7 atm in contact with a liquid nitrogen reservoir [13]. The cryogenic gas cell had a length of $9.0 \mathrm{~cm}$ with $4 \mu \mathrm{m}$ Havar entrance and exit windows of $1.9 \mathrm{~cm}$ diameter. A magnetic stirring system was operated to provide circulation of the gas. The experimental setup is schematically shown in Fig. 1. An aluminum catcher foil, placed after the hydrogen gas cell, was used to collect the ${ }^{67} \mathrm{Cu}$ nuclei, along with other coproduced nuclides. The Al catcher foil was mounted on a rectangular aluminum target frame with a $1.27 \mathrm{~cm}$ diameter hole.

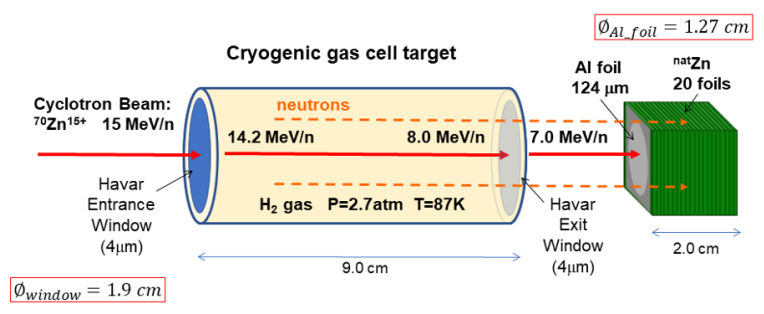

Fig. 1. (Color online) Schematic diagram of the irradiation setup. The energies of the beam are listed as it passes through the entrance window, the gas and the exit window (thick arrows). The dashed arrows represent the neutrons produced via the interactions of the ${ }^{70} \mathrm{Zn}$ beam with the hydrogen gas and the $\mathrm{Al}$ catcher [1].

\subsection{Irradiation}

The irradiation time was $6.5 \mathrm{~h}$ with a beam current of $0.19(5)$ pnA (particle nA). The current was periodically monitored at a Faraday cup positioned after the Cyclotron exit and was nearly constant (within $13 \%$ ). The measurement of the current after the gas cell was performed by a second Faraday cup mounted on the same target ladder as the $\mathrm{Al}$ catcher frame. The measurement of the beam current at this location was 8.0 $\mathrm{nA}$ of ${ }^{70} \mathrm{Zn}(7.0 \mathrm{MeV} /$ nucleon $)$ at an average charge state of $26^{+}$. The average equilibrium charge-state was calculated with LISE++ Physical Calculator [14] based on model of G. Schiwietz et al. [15] for ions with energies lower than $15 \mathrm{MeV} /$ nucleon. The beam current was determined considering the beam charge state before and after the gas cell and the transmission ratio between the two Faradays cups. As electron suppression of the Faraday cups was not performed in the present experiment, the sputtering of electrons from the side of the Faraday cup hit by the heavy-ion beam resulted in an increase of the measured positive current. A suppression test was performed for the Faraday cup positioned after the gas target using a ${ }^{21} \mathrm{Ne}$ beam at $28 \mathrm{MeV} /$ nucleon and a decrease of $40 \%$ of the beam intensity was observed. The beam intensity for that Faraday cup was corrected by this factor and the uncertainty due to this correction was estimated at about $20 \%$. Considering all uncertainties, beam intensity, 13\%; secondary emission electrons suppression, $20 \%$; charge state, $15 \%$; and transmission ratio, $4 \%$; the total uncertainty for the beam current is $28 \%$.

\subsection{Off-line $\gamma$-spectra measurement}

After $36.5 \mathrm{~h}$ from the end of the irradiation, the Al catcher foil was placed in front of a high-purity germanium (HPGe) detector for off-line $\gamma$-ray analysis. The foil was placed at a distance of $\mathrm{d}=17.2(10) \mathrm{mm}$ from the end cap of the detector. The dead time of the counting system was about $2-3 \%$, and the energy resolution of the detector system was 2.5-4.0 keV (FWHM) for the peaks of interest. The energy calibration was performed with a ${ }^{152} \mathrm{Eu}$ source and adjusted by using known $\gamma$-rays obtained in the spectra. The absolute photopeak efficiencies for the actual source-detector geometry were obtained with the Monte Carlo codes GEANT4 [16] and EGSnrc [17]. The

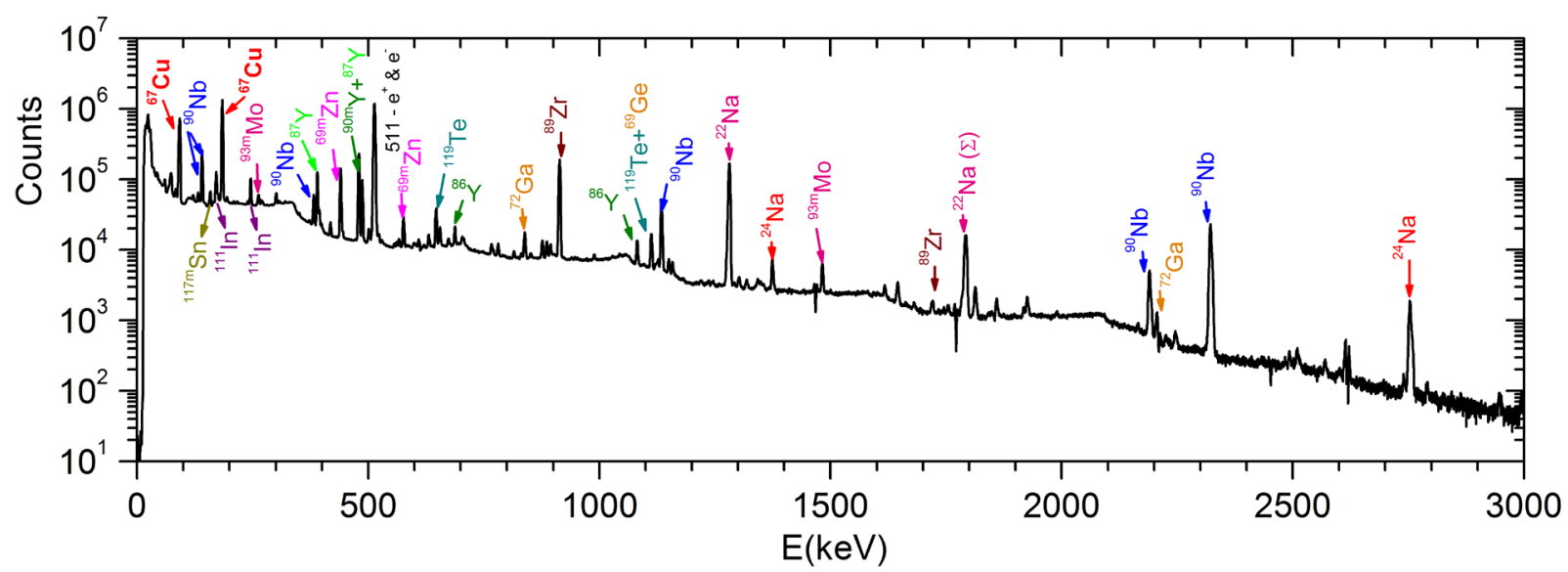

Fig. 2. Al catcher foil background-subtracted $\gamma$-ray spectrum after the interaction of a ${ }^{70} \mathrm{Zn}(15 \mathrm{MeV} /$ nucleon $)$ beam with the hydrogen gas cell after $36.5 \mathrm{~h}$ from the end of the irradiation. The peaks are labelled with the corresponding radionuclides. The peak marked ${ }^{22} \mathrm{Na}(\Sigma)$ is the sum peak induced by the simultaneous detection of the $\beta$ delayed $\gamma$-ray $(1275 \mathrm{keV})$ and $511 \mathrm{keV} \gamma$-ray from e $\mathrm{e}^{+} \& \mathrm{e}^{-}$ annihilation. 
uncertainty assumed for the efficiency simulation results is $10 \%$. The spectrum of the room background was measured for $67.3 \mathrm{~h}$. The background-subtracted $\gamma$-ray spectrum obtained during an accumulation period of $68 \mathrm{~h}$, starting $36.5 \mathrm{~h}$ after the end of the irradiation, is presented in Fig. 2.

\section{Analysis and results}

\subsection{Activities}

The activities at the End of Bombardment $\left(A_{E O B}\right)$ were calculated with the equation:

$$
A_{E O B}=\frac{C}{\varepsilon(E) t_{L} I_{\gamma}} e^{\lambda t_{d}} \frac{\lambda t_{R}}{\left(1-e^{-\lambda t_{R}}\right)}
$$

where $\mathrm{C}$ is the number of counts, $\varepsilon(\mathrm{E})$ the detector efficiency, $I_{\gamma}$ the intensity of the $\gamma$-line of interest, $\lambda$ the decay constant of the radionuclide, $t_{L}$ and $t_{R}$ are respectively the live time and real time of the $\gamma$ spectroscopy measurement, and $t_{d}$ is the decay time elapsed from the end of irradiation to the start of the $\gamma$ spectroscopy measurement. The activities at the end of bombardment ( $\left.\mathrm{A}_{\mathrm{EOB}}\right)$ as well as the activities considering a beam intensity of $1 \mathrm{pnA}$ and an irradiation time of $1 \mathrm{~h}$, the so-called $1 \mathrm{pnA}-1 \mathrm{~h}$ activity $\left(\mathrm{H}_{\mathrm{EOB}}\right)$, are presented in Table 1. Other corrections: (i) $5.3 \%$ for the number of ${ }^{67} \mathrm{Cu}$ produced at gas target and not collected by the Al foil due to the solid angle, obtained from the GEANT4 [16] Monte Carlo simulation (see section 3.2 for details); and (ii) the $0.97 \%$ dead time correction for the data acquisition, already accounted for in equation (1) by the ratio $t_{R} / t_{L}$, were applied to the activity values. The $A_{\text {EOB }}$ uncertainties include the peak area fit, the background determination and the HPGe detector efficiency uncertainties. For the $\mathrm{H}_{\mathrm{EOB}}$ values the beam current uncertainty is incorporated. For each radionuclide produced, Table 1 shows the decay mode, the half-life, and the respective gamma-ray energies $\left(\mathrm{E}_{\gamma}\right)$ and intensities $\left(\mathrm{I}_{\gamma}\right)$ used to obtain the activities. The principal production routes are also presented. At the end of the $6.5 \mathrm{~h}$ of irradiation, the activity of the ${ }^{67} \mathrm{Cu}$ isotope was $2.16(12) \mathrm{kBq}$ and the $1 \mathrm{pnA}-1 \mathrm{~h}$ activity was $1.8(5) \mathrm{kBq} /(\mathrm{pnAh})$. This result includes the Al foil frame contribution. The photopeak associated to $\mathrm{E}_{\gamma}$ doublet at $91.3 \mathrm{keV}$ and $93.3 \mathrm{keV}$ is not resolved in the present measurement. The results were calculated as the weighted average of the activities obtained from the peaks at $\mathrm{E}_{\gamma}=(91.3+93.3) \mathrm{keV}$ and $184.6 \mathrm{keV}$ using the respective intensities (relative to 100 beta decays of the parent $)$ of $\mathrm{I}_{\gamma}=(7.0 \%+16.1 \%)$ and $48.7 \%$ as weighting factors. Note that these photopeaks are pure, i.e., the contribution of $\gamma$-ray transitions is just from ${ }^{67} \mathrm{Cu}$ decay. Other $\gamma$-rays are too weak to be used for analysis and some are contaminated by impurities. The photopeak at $209 \mathrm{keV}$ has contributions from ${ }^{79} \mathrm{Kr}\left(\mathrm{T}_{1 / 2}=35.04 \mathrm{~h}, \mathrm{E}_{\gamma}\right.$ $\left.=208.5 \mathrm{keV}, \mathrm{I}_{\gamma}=0.797 \%\right)$. The next photopeak at 300 $\mathrm{keV}$ is multiply contaminated with ${ }^{79} \mathrm{Kr}\left(\mathrm{T}_{1 / 2}=35.04 \mathrm{~h}\right.$, $\left.\mathrm{E}_{\gamma}=299.5 \mathrm{keV}, \mathrm{I}_{\gamma}=1.54 \%\right),{ }^{101} \mathrm{Pd}\left(\mathrm{T}_{1 / 2}=8.47 \mathrm{~h}, \mathrm{E}_{\gamma}=\right.$ $\left.296.3 \mathrm{keV}, \mathrm{I}_{\gamma}=19 \%\right)$ and ${ }^{73} \mathrm{Ga}\left(\mathrm{T}_{1 / 2}=4.86 \mathrm{~h}, \mathrm{E}_{\gamma}=297.3\right.$ $\left.\mathrm{keV}, \mathrm{I}_{\gamma}=79.8 \%\right)$. Finally, the peak at $393 \mathrm{keV}$ also contains ${ }^{88} \mathrm{Zr}\left(\mathrm{T}_{1 / 2}=83.4 \mathrm{~d}, \mathrm{E}_{\gamma}=392.9 \mathrm{keV}, \mathrm{I}_{\gamma}=97 \%\right)$.
${ }^{79} \mathrm{Kr}$ and ${ }^{88} \mathrm{Zn}$ are produced from the beam interaction with $\mathrm{Al}$ foil, while ${ }^{101} \mathrm{Pd}$ and ${ }^{73} \mathrm{Ga}$ are from beam interaction with $\mathrm{Cr}$ from Havar windows.

Another important radionuclide observed that is produced by the reaction ${ }^{70} \mathrm{Zn}+\mathrm{p}$ is the ${ }^{69 \mathrm{~m}} \mathrm{Zn}\left(\mathrm{T}_{1 / 2}=13.8\right.$ $\mathrm{h})$, the activity of which at the end of the irradiation is $2.55(26) \mathrm{kBq}$, and the $1 \mathrm{pnA}-1 \mathrm{~h}$ activity is $2.2(6)$ $\mathrm{kBq} /(\mathrm{pnAh})$. The transition $\mathrm{E} \gamma=438.6 \mathrm{keV}$ ( $\mathrm{I}_{\gamma}=94.77 \%$ ) was used in the analysis of this isotope. As the ${ }^{69 \mathrm{~m}} \mathrm{Zn}$ half-live is 4.5 smaller than the half-life of ${ }^{67} \mathrm{Cu}$, its activity can be reduced by an appropriate cooling period.

Other coproduced radionuclides are due to the beam interaction on the $\mathrm{Al}$ catcher foil and on the Havar windows. The radionuclides ${ }^{86} \mathrm{Y},{ }^{87} \mathrm{Y},{ }^{89} \mathrm{Zr},{ }^{90} \mathrm{Nb}$ and ${ }^{93 \mathrm{~m}} \mathrm{Mo}$ are fusion-evaporation products of the reaction ${ }^{70} \mathrm{Zn}+{ }^{27} \mathrm{Al}$ (with the beam of ${ }^{70} \mathrm{Zn}$ entering the $\mathrm{Al}$ catcher at 7.0 MeV/nucleon), of which ${ }^{90} \mathrm{Nb}$ is the main impurity with an $\mathrm{A}_{\mathrm{EOB}}=2.38(23) \mathrm{kBq}$. The heavier radionuclides ${ }^{111} \mathrm{In},{ }^{117 \mathrm{~m}} \mathrm{Sn}$ and ${ }^{119} \mathrm{Te}$ are residues of the reaction of the ${ }^{70} \mathrm{Zn}$ beam with the constituents of the Havar alloy (Co $42.0 \%$, Mo 2.2\%, Cr 19.5\%, Mg 1.6\%, Ni 12.7\%, C $0.2 \%, \mathrm{~W} 2.7 \%$, and Fe balance) of the gas-cell windows.

Table 1 presents the activities at the end of bombardment for ${ }^{67} \mathrm{Cu}$ and the most intense radioimpurities, together with some important parameters used in the analysis.

Cross section simulations using the code PACE4 [18] for incident ${ }^{70} \mathrm{Zn}$ at $7.0 \mathrm{MeV} /$ nucleon on ${ }^{27} \mathrm{Al},{ }^{59} \mathrm{Co}$ and ${ }^{52} \mathrm{Cr}$ were performed to support the impurity analysis. The ${ }^{90} \mathrm{Nb}$ production presented a high cross section of $\sigma\left({ }^{90} \mathrm{Nb}\right)=25 \mathrm{mb}$ from the beam interaction with ${ }^{27} \mathrm{Al}$. Considering the beam range on $\mathrm{Al}$ foil is $65 \mu \mathrm{m}$, the main contribution is from the reaction ${ }^{27} \mathrm{Al}\left({ }^{70} \mathrm{Zn}, \mathrm{x}\right){ }^{90} \mathrm{Nb}$. The results for Havar foil, considering the beam interaction with the most abundante isotopes of $\mathrm{Co}$ and $\mathrm{Cr}$ are, $\sigma\left({ }^{59} \mathrm{Co}\right)=1.7 \mathrm{mb}$ and $\sigma\left({ }^{52} \mathrm{Cr}\right)=1.2 \mathrm{mb}$. The other radioimpurities shown in the table are at least a factor 2.5 smaller than ${ }^{67} \mathrm{Cu} \mathrm{A}_{\mathrm{EOB}}$ with all of them mostly produced by the ${ }^{70} \mathrm{Zn}+{ }^{27} \mathrm{Al}$ reactions.

The radionuclides produced in the proton induced reactions on ${ }^{70} \mathrm{Zn}$ in the incident energy range of $7.8-$ 14.3 MeV/nucleon (beam energy at the end of the gas cell and after first Havar window) are ${ }^{67} \mathrm{Cu}$ and ${ }^{69 m} \mathrm{Zn}$.

It is worth mentioning that ${ }^{67} \mathrm{Cu}$ and ${ }^{67} \mathrm{Ga}$ are the beta minus and electron capture parents of ${ }^{67} \mathrm{Zn}$ daughter, using almost the same set of gamma decays, which can make more difficult their separation through gamma ray analysis (moreover they also have similar half-lives, $78.3 \mathrm{~h}$ for ${ }^{67} \mathrm{Ga}$ and $61.9 \mathrm{~h}$ for ${ }^{67} \mathrm{Cu}$ ). However, at this energy range the production of ${ }^{67} \mathrm{Ga}$ is suppressed $\left({ }^{70} \mathrm{Zn}(\mathrm{p}, 4 \mathrm{n}){ }^{67} \mathrm{Ga} \mathrm{E}_{\text {threshold }}=28.081 \mathrm{MeV}\right)$. The others usual radioimpurities coproduced with ${ }^{67} \mathrm{Cu}$, as ${ }^{61,64} \mathrm{Cu}$, ${ }^{66} \mathrm{Ga},{ }^{65} \mathrm{Zn}$ are also excluded due to high energy threshold.

\subsection{Cross sections}

The cross sections up to proton energy of $17 \mathrm{MeV}$ for 
Table 1. Activities obtained in the present work as defined in the text (last two columns) for ${ }^{67} \mathrm{Cu}$ and the main contaminants, the radioactive decay data of the respective radionuclides, and the contributing reactions. The listed $\gamma$ rays were used for the activity calculation.

\begin{tabular}{|c|c|c|c|c|c|c|c|}
\hline $\begin{array}{l}\text { Radio- } \\
\text { nuclide }\end{array}$ & $\begin{array}{l}\text { Decay } \\
\text { mode }\end{array}$ & Half life & $\begin{array}{c}\mathrm{E} \gamma \\
(\mathrm{keV})\end{array}$ & $\mathrm{I} \gamma(\%)$ & $\begin{array}{c}\text { Principal } \\
\text { Production routes }\end{array}$ & $\begin{array}{c}\text { AEOB }_{\text {EOB }} \\
(\boldsymbol{k B q})\end{array}$ & 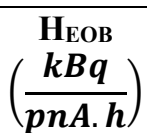 \\
\hline \multirow[t]{3}{*}{${ }^{67} \mathrm{Cu}$} & $\beta^{-}$ & 61.83 h 12 & $91.266(5)$ & $7.0(1)$ & $\mathrm{p}\left({ }^{70} \mathrm{Zn},{ }^{67} \mathrm{Cu}\right) \alpha$ & $2.16(12)$ & $1.8(5)$ \\
\hline & & & $93.311(5)$ & $16.1(2)$ & & & \\
\hline & & & $184.577(10)$ & $48.7(3)$ & & & \\
\hline${ }^{69} \mathrm{~m} \mathbf{Z n}$ & $\mathrm{IT}, \beta^{-}$ & 13.76 h 2 & $438.63(2)$ & $94.77(20)$ & $\mathrm{p}\left({ }^{70} \mathrm{Zn},{ }^{69 \mathrm{~m}} \mathrm{Zn}\right) \mathrm{d}$ & $2.55(26)$ & $2.2(6)$ \\
\hline \multirow[t]{3}{*}{${ }^{90} \mathrm{Nb}$} & $\varepsilon^{+} \beta^{-}$ & 14.60 h 5 & $1129.224(15)$ & $92.7(4)$ & ${ }^{27} \mathrm{Al}\left({ }^{70} \mathrm{Zn},{ }^{90} \mathrm{Nb}\right) \mathrm{x}$ & $2.38(23)$ & $2.0(6)$ \\
\hline & & & $2318.968(10)$ & $82.03(16)$ & ${ }^{59} \mathrm{Co}\left({ }^{70} \mathrm{Zn},{ }^{90} \mathrm{Nb}\right) \mathrm{x}$ & & \\
\hline & & & $2186.242(25)$ & $17.96(16)$ & ${ }^{52} \mathrm{Cr}\left({ }^{70} \mathrm{Zn},{ }^{90} \mathrm{Nb}\right) \mathrm{x}$ & & \\
\hline${ }^{87 m} \mathbf{Y}$ & IT & 13.37 h 3 & $380.79(7)$ & 78 & ${ }^{27} \mathrm{Al}\left({ }^{70} \mathrm{Zn},{ }^{87 \mathrm{~m}} \mathrm{Y}\right) \mathrm{x}$ & $0.87(9)$ & $0.74(22)$ \\
\hline${ }^{89} \mathrm{Zr}$ & $\varepsilon^{+} \beta^{+}$ & 78.41 h 12 & $908.96(4)$ & 100 & ${ }^{27} \mathrm{Al}\left({ }^{70} \mathrm{Zn},{ }^{89} \mathrm{Zr}\right) \mathrm{x}$ & $0.62(6)$ & $0.52(16)$ \\
\hline${ }^{22} \mathrm{Na}$ & $\varepsilon^{+} \beta^{+}$ & 2.6019 y 4 & $1.27453(2)$ & $99.944(14)$ & $\begin{array}{c}{ }^{27} \mathrm{Al}\left({ }^{70} \mathrm{Zn},{ }^{22} \mathrm{Na}\right) \mathrm{x} \\
{ }^{27} \mathrm{Al}(\mathrm{n}, \mathrm{x})^{22} \mathrm{Na}\end{array}$ & $0.46(5)$ & $0.41(12)$ \\
\hline \multirow[t]{6}{*}{${ }^{86} \mathbf{Y}$} & $\varepsilon^{+} \beta^{+}$ & 14.74 h 2 & $627.72(10)$ & $32.6(10)$ & ${ }^{27} \mathrm{Al}\left({ }^{70} \mathrm{Zn},{ }^{86} \mathrm{Y}\right) \mathrm{x}$ & $0.38(4)$ & $0.32(10)$ \\
\hline & & & $1153.01(4)$ & $30.5(9)$ & ${ }^{59} \mathrm{Co}\left({ }^{70} \mathrm{Zn},{ }^{86} \mathrm{Y}\right) \mathrm{x}$ & & \\
\hline & & & 1920.72(13) & $20.8(7)$ & ${ }^{52} \mathrm{Cr}\left({ }^{70} \mathrm{Zn},{ }^{86} \mathrm{Y}\right) \mathrm{x}$ & & \\
\hline & & & $1854.38(13)$ & $17.2(5)$ & & & \\
\hline & & & $443.14(9)$ & $16.9(5)$ & & & \\
\hline & & & 703.34(10) & $15.4(4)$ & & & \\
\hline \multirow[t]{3}{*}{${ }^{87} \mathbf{Y}$} & $\varepsilon^{+} \beta^{+}$ & 79.8 h 3 & $484.805(5)$ & $89.7(3)$ & ${ }^{27} \mathrm{Al}\left({ }^{70} \mathrm{Zn},{ }^{87} \mathrm{Y}\right) \mathrm{x}$ & $0.167(16)$ & $0.15(4)$ \\
\hline & & & 388.531(3) & 82 & ${ }^{59} \mathrm{Co}\left({ }^{70} \mathrm{Zn},{ }^{87} \mathrm{Y}\right) \mathrm{x}$ & & \\
\hline & & & & & ${ }^{52} \mathrm{Cr}\left({ }^{70} \mathrm{Zn},{ }^{87} \mathrm{Y}\right) \mathrm{x}$ & & \\
\hline
\end{tabular}

${ }^{70} \mathrm{Zn}(\mathrm{p}, \mathrm{x}){ }^{67} \mathrm{Cu}$ and ${ }^{70} \mathrm{Zn}(\mathrm{p}, \mathrm{x}){ }^{69 \mathrm{~m}} \mathrm{Zn}$ from [19-22] are presented in Fig. 3. For ${ }^{67} \mathrm{Cu}$ production there are the experimental data obtained by Levkovskij et al. [19] (triangle) and Kastleiner et al. [20] (square) in comparison with the IAEA [21] recommended cross section extracted from a fit of these two data sets (dashdot line). The experimental data for ${ }^{69 \mathrm{~m}} \mathrm{Zn}$ production from Levkovskij et al. [19] are also represented (circle).

The theoretical predictions from the TENDL-2019 library [22], based on the TALYS code [28], are also represented (dot line). These predictions did not reproduce well the experimental data, for ${ }^{67} \mathrm{Cu}$ the values are systematically higher and for ${ }^{69 \mathrm{~m}} \mathrm{Zn}$ the results underestimate the experimental cross sections in the region of 12-14 MeV. The value adopted by Levkovskij et al. [19] in the monitor reaction ${ }^{\text {nat }} \mathrm{Mo}(\mathrm{p}, \mathrm{x})^{96} \mathrm{Tc}$ cross section at $30 \mathrm{MeV}$ was $250 \mathrm{mb}$ while more recent cross section evaluation recommended by IAEA [21] is $192.82 \mathrm{mb}$. The data represented are normalized by a factor 0.77 to take into account the correction in the monitor cross section. The energy region of interest for the present work is within the two dash-dot bars in the graph.

The present measurement is a thick target inverse kinematics measurement as the $15 \mathrm{MeV} /$ nucleon ${ }^{70} \mathrm{Zn}$ beam impinging on $2.7 \mathrm{~atm}$ gas target results in $\Delta \mathrm{E} / \mathrm{E}=$
0.45. To take that into account, a Monte Carlo simulation with the GEANT4 package [16] was performed. The cross sections used for the simulations were the IAEA recommended for ${ }^{67} \mathrm{Cu}$ and the interpolation of the corrected experimental data from Levkovskij et al. [19] for ${ }^{69 \mathrm{~m}} \mathrm{Zn}$. The beam energy loss and the position on gas target where the reactions $\mathrm{p}\left({ }^{70} \mathrm{Zn},{ }^{67} \mathrm{Cu}\right) \alpha$ and $\mathrm{p}\left({ }^{70} \mathrm{Zn},{ }^{69 \mathrm{~m}} \mathrm{Zn}\right) \mathrm{d}$ occur according to GEANT4 simulation are presented in Fig. 4. The beam energy is represented on the $y$ axis as a function of the target position where the reaction occurs on the $\mathrm{x}$ axis. Most of the events are concentrated at the beginning of the target as expected. The simulation results have shown that the number of ${ }^{67} \mathrm{Cu}$ nuclei produced at the gas target differs by $5.3 \%$ from that collected in the $\mathrm{Al}$ foil. This result is due to the solid angle and scattering on the gas target.

The experimental energy loss weighted average cross sections in units $\left[\mathrm{cm}^{3}\right]$ were calculated from:

$$
\langle\sigma R\rangle_{E x p}=\frac{A_{E O B} m_{\text {target }} m_{u} e}{\rho_{\text {target }} \varphi\left(1-e^{\left.-\lambda t_{\text {irr }}\right)}\right.}
$$

where $\rho_{\text {target }}$ is the density of the target $\left[\mathrm{g} / \mathrm{cm}^{3}\right], \varphi$ is the beam intensity [pA], $t_{i r r}$ is the irradiation time [s], $m_{\text {target }}$ is the atomic mass of the target $[\mathrm{u}], \mathrm{m}_{\mathrm{u}}$ atomic mass constant $[\mathrm{g} / \mathrm{u}]$ and $\mathrm{e}$ is the elementary charge $[\mathrm{C}]$. 


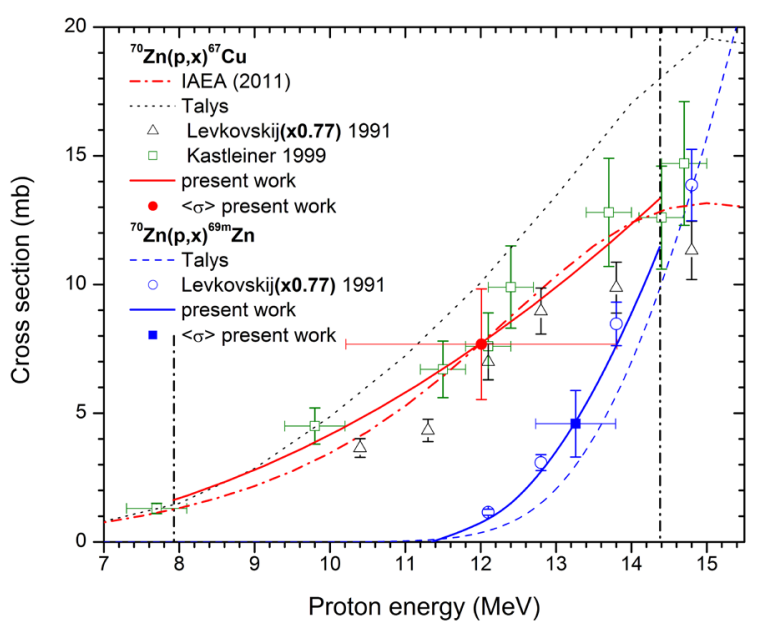

Fig. 3. (Color online) Experimental cross sections for ${ }^{70} \mathrm{Zn}(\mathrm{p}, \mathrm{x}){ }^{67} \mathrm{Cu}$ and ${ }^{70} \mathrm{Zn}(\mathrm{p}, \mathrm{x})^{69 \mathrm{~m}} \mathrm{Zn}$ from Levkovskij et al. [19] and Kastleiner et al. [20] in comparison with theoretical predictions from the TENDL-2019 library [22], based on the TALYS code and the IEAE [21] recommended cross section for ${ }^{67} \mathrm{Cu}$. The present work results for the average cross section associated with ${ }^{67} \mathrm{Cu}$ and ${ }^{69 \mathrm{~m}} \mathrm{Zn}$ production and the estimated cross section for the region are also presented.

\section{$A_{E O B}$ is expressed in $[\mathrm{Bq}]$ and $\lambda$ in $\left[\mathrm{s}^{-1}\right]$.}

The cross section can be estimated assuming a parametrization as:

$$
\sigma=p_{0}\left(E-E_{\min }\right)^{2}
$$

where $E_{\text {min }}$ is the minimum energy that the reaction occurs and $p_{0}$ is a parameter to be extracted from experimental data. As the reaction $\mathrm{p}\left({ }^{70} \mathrm{Zn},{ }^{67} \mathrm{Cu}\right) \alpha$ has zero threshold energy, the minimum energy is associated with the Coulomb barrier potential, $E_{\text {min }} .=$ 4.4 MeV. Considering the reaction $\mathrm{p}\left({ }^{70} \mathrm{Zn},{ }^{69 \mathrm{~m}} \mathrm{Zn}\right) \mathrm{d}$, it has a $Q_{\text {value }}=-7.5 \mathrm{MeV}$, thus the cross-section is expected to decrease rapidly with decreasing beam energy. The available experimental data for this reaction indicates a minimum energy of $E_{\text {min }}=11.2 \mathrm{MeV}$ (see Fig. 3), that value was adopted here. The energy loss weighted crosssection is given by the following expression:

$$
\langle\sigma R\rangle=\int_{E_{\min }}^{E_{\max }} \frac{\sigma(E)}{\frac{d E}{d x}} d E
$$

where $\frac{d E}{d x}$ is the stopping power. From equation (2) and (3) it is possible to extract the parameter $p_{0}$ using the experimental results $\langle\sigma R\rangle_{E x p}$ and the $\frac{d E}{d x}$ obtained from the SRIM code [23] by:

$$
p_{0}=\frac{\langle\sigma R\rangle_{E x p}}{\int_{E_{\min }}^{E_{\max } \frac{\left(E-E_{0}\right)^{2}}{\frac{d E}{d x}} d E}}
$$

The most probable energy where reactions occur was determined using the mean value theorem for the integral of equation (4).

$$
\langle\sigma R\rangle_{E x p}=\left(E_{\max }-E_{\min }\right) \frac{p_{0}\left(\langle E\rangle-E_{\min }\right)^{2}}{\frac{d E}{d x}(\langle E\rangle)}
$$

Table 2 presents the energy loss weighted average cross sections and the parameter $p_{0}$, equation (2) and (5), obtained from the experimental results. The respective average energy $\langle E\rangle$ from equation (6) and the average cross section $\langle\sigma\rangle$ are also shown in Table 2 and Figure 3. The estimated parametrized cross section is represented in Figure 3 with solid lines. The $\langle E\rangle$ uncertainty was obtained from the propagation of $\langle\sigma R\rangle_{E x p}$ and $p_{0}$ uncertainties.

Note the results for ${ }^{67} \mathrm{Cu}$ production are in good agreement with experimental cross section from Kastleiner et al. [20]. For ${ }^{69 \mathrm{~m}} \mathrm{Zn}$, the parametrized cross section describes well the experimental values from Levkovskij et al. [19] and the average cross section considering the errors bars agrees with the experimental data. Notice that the ansatz, equation (3), could be easily improved if one or more experimental points are added. In fact, in that case the parameters $\mathrm{E}_{\min }$ and $p_{0}$ could be left free. However, the goal is to test a new method to obtain cross-sections using thick targets. The approach, once is suitably adjusted to the experimental case, can be very powerful for instance for the data of ${ }^{99} \mathrm{Mo}$ production [24] where there are three experimental points. A similar methodology is used by $\mathrm{P}$. Tan et al. [25] for sub-barrier fusion reactions, but using derivatives of equation (4) instead. The approach here does not require derivatives and can be easily extended to those reactions.

Table 2: The energy loss weighted average cross section and the parameter $p_{0}$ obtained from experimental results, and the respective average energy and cross section for ${ }^{67} \mathrm{Cu}$ and ${ }^{69 \mathrm{~m}} \mathrm{Zn}$.

\begin{tabular}{ccccc}
\hline & $\begin{array}{c}\langle\boldsymbol{\sigma} R\rangle_{\boldsymbol{E x p}} \\
(\boldsymbol{m b} \cdot \boldsymbol{c m})\end{array}$ & $\begin{array}{c}\boldsymbol{p}_{\mathbf{0}} \\
\left(\boldsymbol{m b} / \mathrm{MeV}^{2}\right)\end{array}$ & $\begin{array}{c}\langle\boldsymbol{E}\rangle \\
(\mathrm{MeV})\end{array}$ & $\begin{array}{c}\langle\boldsymbol{\sigma}\rangle \\
(\boldsymbol{m b})\end{array}$ \\
\hline${ }^{{ }^{67} \mathrm{Cu}}$ & $53(15)$ & $0.14(4)$ & $12.0(18)$ & $7.7(21)$ \\
${ }^{69 m} \mathrm{Zn}$ & $16(4)$ & $1.3(4)$ & $13.3(5)$ & $4.6(13)$ \\
\hline
\end{tabular}

\subsection{The thick target yield in inverse kinematics}

In Nuclear Data of radioactive isotopes, besides the cross section, the Thick Target Yield (TTY) is an important quantity to be determined. The yield is usually defined as the ratio of the nuclei produced in the reaction over the number of incident particles. In the yield for a radionuclide production, the number of radioactive nuclei is associated to the activity and the number of incident particles to the beam charge. The Thick Target Yield (TTY) is defined for a determined macroscopic energy loss and it is given by the equation [26]:

$$
T T Y\left[\frac{B q}{C}\right]=\frac{N_{A} \lambda \rho_{\text {target }}}{Z_{\text {beam }} e M_{\text {target }}}\langle\sigma R\rangle
$$

where $N_{A}$ is Avogadro's number $\left[\mathrm{mol}^{-1}\right], Z_{\text {beam }}$ is the charge of the fully ionized projectile, $e$ is the elementary charge [C] and $M_{\text {target }}$ is the molar mass of the target $[\mathrm{g} / \mathrm{mol}]$. The respective units for the following quantities are: $\lambda\left[\mathrm{s}^{-1}\right], \rho\left[\mathrm{g} / \mathrm{cm}^{3}\right]$ and $\langle\sigma R\rangle\left[\mathrm{cm}^{3}\right]$.

The TTY can be determined experimentally from the $A_{E O B}$ from the following equation:

$$
T T Y^{\exp }\left[E_{\min } \rightarrow E_{\text {max }}\right]=\frac{A_{E O B}}{I} \frac{\lambda}{\left(1-e^{\left.-\lambda t_{i r r}\right)}\right.}
$$



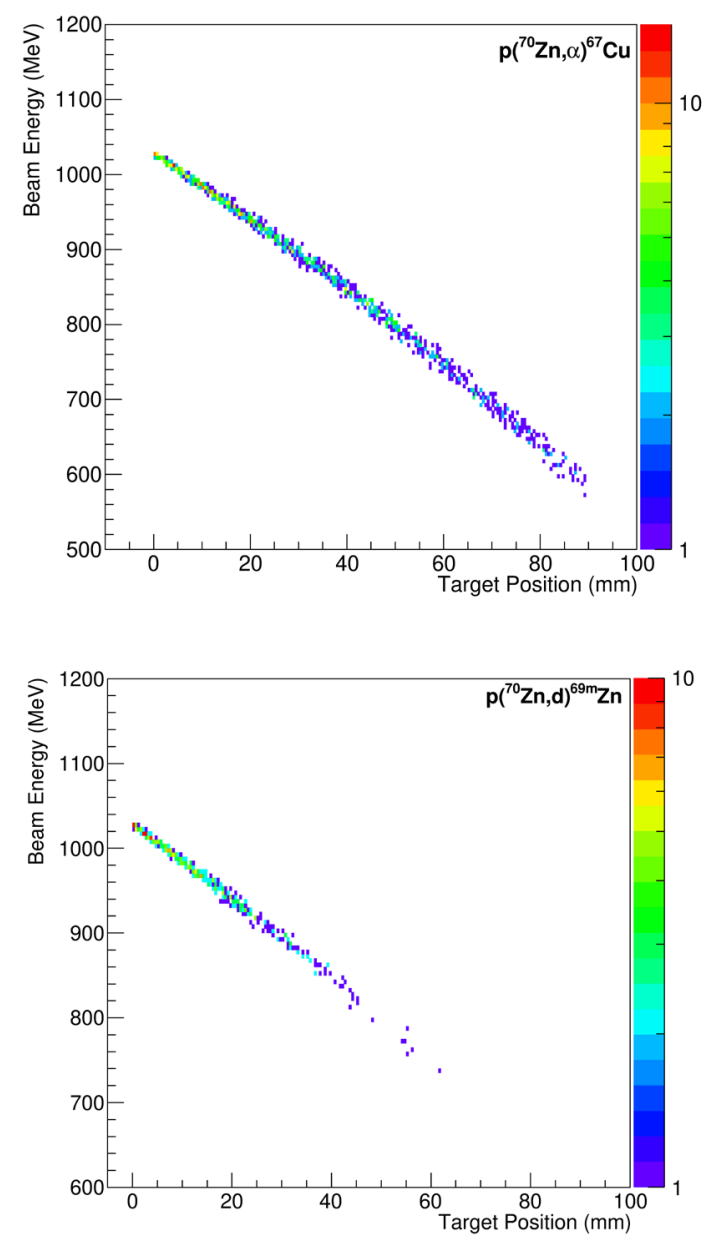

Fig. 4. (Color online) Beam energy as a function of reaction position on the gas target for the reactions $\mathrm{p}\left({ }^{70} \mathrm{Zn},{ }^{67} \mathrm{Cu}\right) \alpha$ (upper part) and $\mathrm{p}\left({ }^{70} \mathrm{Zn},{ }^{69 \mathrm{~m}} \mathrm{Zn}\right) \alpha$ (lower part) according to GEANT simulation [16].

where $\mathrm{I}$ is the beam intensity [A]. The $T T Y_{\text {inv }}^{\text {calc }}$ predicted and the $T T Y_{\text {inv }}^{\text {exp }}$ experimentally determined, seen in Table 3, are in good agreement considering the uncertainties. There are several programs and tables in the literature with information about TTY but in most cases, they are forward kinematics reactions [21]. Although in inverse kinematics the TTY definition does not change, the stopping power $\frac{d E}{d x}$ does. The conversion from $T T Y_{\text {inv }}$ to $T T Y_{\text {for }}$ can be done, as described in the work of M. Aikawa et al. [27], with the ratio $R(E)$ between the differential yields:

$$
\begin{array}{r}
R(E)=\frac{Z_{\text {target }}}{Z_{\text {beam }}} \frac{M_{\text {beam }}}{M_{\text {target }}} \frac{\rho_{\text {target }}}{\rho_{\text {beam }}} \frac{\frac{d E}{d x_{\text {inv }}}}{\frac{d E}{d x} \text { for }} \\
T T Y_{\text {for }}(E) \cong R(E) T T Y_{\text {inv }}(E)
\end{array}
$$

For the reaction ${ }^{70} \mathrm{Zn}+\mathrm{p}$ in the energy range $\left(\mathrm{E}_{\mathrm{c} . \mathrm{m} .}=7.8-\right.$ $14.3 \mathrm{MeV}$ ) the ratio is $R(E) \cong 18.4$. In this way the experimental TTY was converted to forward kinematics. The results for the ${ }^{67} \mathrm{Cu}$ are also in good agreement with the integral yields recommended by IAEA [21], $Y\left(E_{\max }\right)-Y\left(E_{\min }\right)$ as seen in Table 3.
Table 3: The thick target yield in inverse kinematics determined from cross section and stopping power $T T Y_{\text {inv }}^{\text {calc }}$, the thick target yield in inverse kinematics determined with the experimental activities and beam current $T T Y_{\text {inv }}^{\text {exp }}$, the conversion of the experimental TTY to forward kinematics $T T Y_{\text {for }}^{\text {exp }}$ and the integral yield, $Y\left(E_{\text {max }}\right)-$

\begin{tabular}{|c|c|c|c|c|}
\hline & $\begin{array}{l}T T Y_{\text {inv }}^{\text {calc }} \\
(\boldsymbol{G B q} / \boldsymbol{C})\end{array}$ & $\begin{array}{l}T T Y_{i n v}^{\exp } \\
(G B q / C)\end{array}$ & $\begin{array}{c}T T Y_{\text {for }}^{\exp } \\
(\boldsymbol{G B q} / \boldsymbol{C})\end{array}$ & $\begin{array}{l}Y\left(E_{\max }\right) \\
-\boldsymbol{Y}\left(\boldsymbol{E}_{\min }\right) \\
(\boldsymbol{G B q} / \boldsymbol{C})\end{array}$ \\
\hline${ }^{67} \mathrm{Cu}$ & $0.0161(16)$ & $0.021(5)$ & $0.38(10)$ & 0.314 \\
\hline${ }^{69 m} \mathrm{Zn}$ & $0.0214(21)$ & $0.027(7)$ & $0.50(14)$ & \\
\hline
\end{tabular}
$Y\left(E_{\min }\right)$, recommended by IEAE [21].

\subsection{Use of the neutrons from the primary reaction for secondary isotope production}

A test using the forward-focused neutrons from the primary reaction to irradiate ${ }^{\text {nat }} \mathrm{Zn}$ in order to produce ${ }^{67} \mathrm{Cu}$ was also performed. The interaction of ${ }^{70} \mathrm{Zn}$ beam at $15 \mathrm{MeV} /$ nucleon with the proton target produces about 1.6 neutrons per reaction, as calculated with the code TALYS [28]. These neutrons are kinematically focused in the forward direction and can be used for further radioisotope production. In the present experiment, a block of twenty $25.4 \times 25.4 \mathrm{~mm}^{2}$ foils of nat $\mathrm{Zn}$ with $1 \mathrm{~mm}$ thickness was placed behind the $\mathrm{Al}$ catcher (Fig. 1).

The $\gamma$-ray spectrum obtained during an accumulation period of $40 \mathrm{~h}$, starting $7.5 \mathrm{~d}$ after the end of the irradiation, is presented in Fig. 5 in comparison with the background spectrum normalized for the measurement time. Note that both spectra are identical, except for the low energy region that is zoomed in the bottom panel of Fig. 5. It is possible to observe that the source spectrum is lower than the background spectrum with this difference increasing for lower energies, this being due to the shielding effect of the source on the background radiation. On the other hand, the photopeak associated to $185 \mathrm{keV}$ energy presents an enhancement. The background contribution is associated to ${ }^{226} \mathrm{Ra}\left(\mathrm{T}_{1 / 2}=\right.$ $1600 \mathrm{y}, \mathrm{E}_{\gamma}=186.2 \mathrm{keV}, \mathrm{I}_{\gamma}=3.59 \%$ ) almost coinciding with the ${ }^{67} \mathrm{Cu}$ source $\left(\mathrm{T}_{1 / 2}=61.9 \mathrm{~h}, \mathrm{E}_{\gamma}=184.6 \mathrm{keV}, \mathrm{I} \gamma=\right.$ $48.7 \%$ ). The respective areas for source and background were determined and subtracted. The activity of ${ }^{67} \mathrm{Cu}$ produced by the neutrons from the primary reaction at the end of the $6.5 \mathrm{~h}$ of irradiation was 5.3(8) Bq. Considering the cross sections and isotopic abundance, the main production of ${ }^{67} \mathrm{Cu}$ comes from ${ }^{67} \mathrm{Zn}(\mathrm{n}, \mathrm{p}){ }^{67} \mathrm{Cu}$ reaction [29]. Considering the neutron angular distribution produced from the primary reaction according to CoMD predictions [30-32], the distribution presents a peak around $\theta_{\mathrm{LAB}} \sim 10-20^{\circ}$. The experimental set up for ${ }^{\text {nat }} \mathrm{Zn}$ target covers the angular range $0^{\circ}-3^{\circ}$ which corresponds to collecting of about $2 \%$ of the flux of the neutrons. Other important corrections are the $\gamma$ attenuation in the source of about $50 \%$ and the isotopic natural abundance of ${ }^{67} \mathrm{Zn}, 4.04 \%$. Note that these results are an estimation and more detailed experimental investigation is needed. 

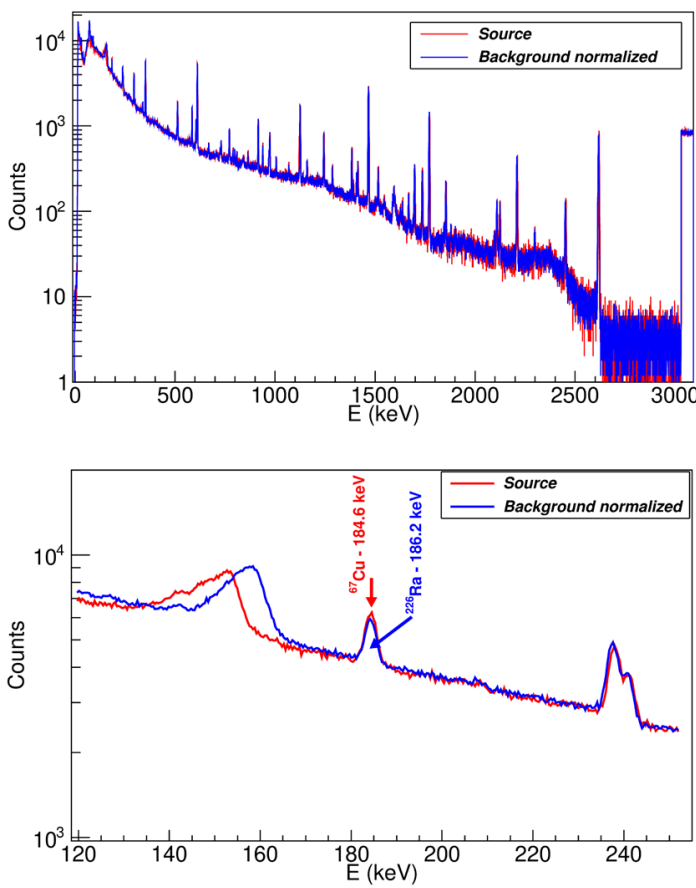

Fig. 5. (Color online) The $\gamma$-ray spectrum obtained during an accumulation period of $40 \mathrm{~h}$, starting $7.5 \mathrm{~d}$ after the end of the irradiation in comparison with the background spectrum normalized for the measurement time.

\section{Conclusion and future plans}

The methodology to produce medically important radionuclides using inverse kinematics [1] has been tested with success. The production of the radionuclide ${ }^{67} \mathrm{Cu}\left(\mathrm{T}_{1 / 2}=62 \mathrm{~h}\right)$ through the reaction of a beam of 15 $\mathrm{MeV} /$ nucleon ${ }^{70} \mathrm{Zn}$ with a cryogenic hydrogen gas target was performed. The activity of the ${ }^{67} \mathrm{Cu}$ isotope after 6.5 $\mathrm{h}$ of irradiation was $\mathrm{A}_{\mathrm{EOB}}=2.16(12) \mathrm{kBq}$ and the $1 \mathrm{pnA}-$ $1 \mathrm{~h}$ activity was $\mathrm{H}_{\mathrm{EOB}}=1.8(5) \mathrm{kBq} /(\mathrm{pnAh})$. The thick target yield in inverse kinematics is $T T Y_{i n v}^{\text {exp }}=0.021(5)$ $(\mathrm{GBq} / \mathrm{C})$ and the correspondent in forward kinematics is $T T Y_{\text {for }}^{\text {exp }}=0.38(10)(\mathrm{GBq} / \mathrm{C})$. The main radioimpurity in the $\mathrm{Al}$ catcher coming from the ${ }^{70} \mathrm{Zn}+\mathrm{p}$ reaction is ${ }^{69 \mathrm{~m}} \mathrm{Zn}\left(\mathrm{T}_{1 / 2}=13.8 \mathrm{~h}\right)$, the activity of which at the end of irradiation is $\mathrm{A}_{\mathrm{EOB}}=2.55(26) \mathrm{kBq}$ and $1 \mathrm{pnA}-1 \mathrm{~h}$ Activity $\mathrm{H}_{\mathrm{EOB}}=2.2(6) \mathrm{kBq} /(\mathrm{pnAh})$. The ${ }^{90} \mathrm{Nb}$ is the main impurity from the beam interaction on the $\mathrm{Al}$ catcher foil with an activity of $\mathrm{A}_{\mathrm{EOB}}=2.38(23) \mathrm{kBq}$. The present successful test indicates the possibility of producing important non-standard radionuclides of high radionuclide purity with the approach of inverse kinematics. The secondary neutrons from the primary reaction were used to irradiate a secondary target of $\mathrm{Zn}$ for further radioisotope production with promising results. The main requirement necessary to achieve the production of activities appropriate for preclinical studies is the availability of high-intensity (particle $\mu \mathrm{A}$ ) heavy-ion primary beams.

\section{Acknowledgements}

We are grateful to the support staff of the Cyclotron Institute for providing the primary beam. Financial support for this work was provided, in part by the U.S. Department of Energy under Grant No. DE-FG03-93ER40773, by the NNSA under grant no de-na0003841 (CENTAUR), and by the Special Account for Research Grants of the National and Kapodistrian University of Athens. M.Y. and P.Z.F. acknowledge the support of the Houston Methodist Research Institute (HMRI).

\section{References}

1. G. A. Souliotis et al., A novel approach to medical radioisotope production using inverse kinematics: A successful production test of the theranostic radionuclide ${ }^{67} \mathrm{Cu}$, Appl. Radiat. Isotopes 149, 89 (2019)

2. G. Hao et al., Copper-67 radioimmunotheranostics for simultaneous immunotherapy and immuno-SPECT, Sci. Rep. 11, 3622 (2021)

3. O. Keinänen,Harnessing et al., ${ }^{64} \mathrm{Cu} /{ }^{67} \mathrm{Cu}$ for a theranostic approach to pretargeted radioimmunotherapy, P. Natl. Acad. Sci. 117, 28316 (2020)

4. N.A. Smith et al., The production, separation, and use of ${ }^{67} \mathrm{Cu}$ for radioimmunotherapy: a review, Appl. Radiat. Isot. 70, 2377-2383 (2012)

5. D. G. Medvedev et al., Development of a large scale production of ${ }^{67} \mathrm{Cu}$ from ${ }^{68} \mathrm{Zn}$ at the high energy proton accelerator: closing the ${ }^{68} \mathrm{Zn}$ cycle, Appl. Radiat. Isot. 70, 423-429 (2008)

6. T. Katabuchi et al., Production of ${ }^{67} \mathrm{Cu}$ via the ${ }^{68} \mathrm{Zn}(p, 2 p){ }^{67} \mathrm{Cu}$ reaction and recovery of ${ }^{68} \mathrm{Zn}$ target. J. Radioanal. Nucl. Chem. 277, 467-470 (2008)

7. G. Pupillo et al., New production cross sections for the theranostic radionuclide ${ }^{67} \mathrm{Cu}$. Nucl. Instrum. Methods Phys. Res. B 415, 41-47 (2018)

8. T. Stoll et al., Excitation functions of proton induced reactions on ${ }^{68} \mathrm{Zn}$ from threshold up to 71 $\mathrm{MeV}$, with specific reference to the production of ${ }^{67} \mathrm{Cu}$, Radiochim. Acta 90, 309-313 (2002)

9. K. Hilgers et al., Cross section measurements of the nuclear reactions ${ }^{n a t} \mathrm{Zn}(d, x)^{64} \mathrm{Cu}$, ${ }^{66} \mathrm{Zn}(d, \alpha){ }^{64} \mathrm{Cu},{ }^{68} \mathrm{Zn}(p, \alpha n){ }^{64} \mathrm{Cu}$ for production of ${ }^{64} \mathrm{Cu}$ and technical developments for small scale production of ${ }^{67} \mathrm{Cu}$ via the ${ }^{70} \mathrm{Zn}(p, \alpha)^{67} \mathrm{Cu}$ process, Appl. Radiat. Isot. 59, 343-351 (2003)

10. D.J. Jamriska SR et al., Activation rates and chemical recovery of ${ }^{67} \mathrm{Cu}$ produced with low energy proton irradiation of enriched ${ }^{67} \mathrm{Zn}$ targets. J. Radioanal. Nucl. Chem. 195, 263-270 (1995)

11. S. Kastleiner et al., Possibility of production of ${ }^{67} \mathrm{Cu}$ at a small-sized cyclotron via the $(p, \alpha)$ reaction on enriched ${ }^{70} \mathrm{Zn}$, Radiochim. Acta 84, 107-110 (1999)

12. R. E. Tribble et al., MARS: a momentum achromat recoil spectrometer, Nucl. Instrum. Methods A 285, 441-446 (1989)

13. J. F. Brinkley et al., Progress in Research. Cyclotron Institute, Texas A\&M University (2002- 
2003), p. V-9 (2009) accessible at: ttp://cyclotron.tamu.edu/ publications.html.

14. O. B. Tarasov, D. Bazin, LISE++: Radioactive beam production with in-flight separators, Nucl. Instrum. Methods Phys. Res. B 266, 4657-4664 (2008)

15. G. Schiwietz et al., Improved charge state formulas, Nucl. Instrum. Methods B 175-177, 125131 (2001)

16. Agostinelli, S., et al., Geant4 a simulation toolkit. Nucl. Instrum. Methods Phys. Res. 506, 250-303 (2003)

17. I. Kawrakow, Accurate condensed history Monte Carlo simulation of electron transport. I. EGSnrc, the new EGS4 version. Med. Phys. 27, 485 (2000). Kawrakow, I., Rogers, D.W.O., NRCC Report PIRS-701, NRC, Ottawa (2003) https://nrccnrc.github.io/EGSnrc/.

18. O.B.Tarasov, D.Bazin Nucl. Instrum. Methods B 266, 4657-4664 (2008); A.Gavron, Phys.Rev. C 21, 230-236 (1980) http://lise.nscl.msu.edu/pace4

19. Levkovskij, Act. Cs. By Protons and Alphas, Moscow, Cross sections of medium mass nuclide activation $(A=40-100)$ by medium energy protons and alpha-particles $(E=10-50 \mathrm{MeV}),(1991)$

20. S. Kastleiner et al., Possibility of Production of ${ }^{67} \mathrm{Cu}$ at a Small-Sized Cyclotron via the $(p, \alpha)$ Reaction on Enriched ${ }^{70} \mathrm{Zn}$, Radiochim. Acta 84, no. 2, 107-110 (1999)

21. INTERNATIONAL ATOMIC ENERGY AGENCY, Nuclear Data for the Production of Therapeutic Radionuclides, Technical Reports Series No. 473, IAEA, Vienna (2012)

22. D. Koning et al., TENDL: Complete Nuclear Data Library for Innovative Nuclear Science and Technology, Nuclear Data Sheets 155, 1-55 (2019)
23. J. Ziegler, J. P. Biersack, M. Ziegler, SRIM: the Stopping and Range of Ions in Matter, http://www.srim.org

24. J. Mabiala et al., Enhanced production of ${ }^{99}$ Mo in inverse kinematics heavy ion reactions, HINPw6 proceedings, EPJ-woc proceedings (article 08003 in this volume).

25. W. P. Tan et al., New Measurement of ${ }^{12} C+{ }^{12} C$ Fusion Reaction at Astrophysical Energies, Phys. Rev. Lett. 124, 192702 (2020)

26. M. Bonardi, The contribution to the nuclear data for biomedical radioisotope production from Milan Cyclotron Laboratory, Data Requirements for Medical Radioisotope Production, INDC(NDS)-193, IAEA, Vienna (1988)

27. M. Aikawa, S. Ebata, S. Imai, Thick-target yields of radioactive targets deduced from inverse kinematics, Nucl. Instrum. Methods Phys. Res. B 353, 1-3, (2015)

28. A.J. Koning, D. Rochman, Modern nuclear data evaluation with the TALYS code system, Nucl. Data Sheets 113 (12), 28412934 (2012) https://tendl.web.psi.ch/tendl_2019/talys.html

29. Z. Karimi et al., Experimental production and theoretical assessment of ${ }^{67} \mathrm{Cu}$ via neutron induced reaction, Annals of Nuclear Energy 133, 665-668 (2019)

30. M. Papa, T. Maruyama, A. Bonasera, Constrained molecular dynamics approach to fermionic systems, Phys. Rev. C 64, 024612 (2001)

31. M. Papa, G. Giuliani and A. Bonasera, Constrained molecular dynamics II: A N-body approach to nuclear systems, J. Comput. Phys. 208, 403-415 (2005)

32. G. Giuliani, H. Zheng, A. Bonasera, The many facets of the (non relativistic) Nuclear Equation of State, Prog. in Part. and Nucl. Phys. 76, 116 (2014) 